\title{
Correspondence
}

\section{Further improvements in the technique of fiberoptic intubation}

To the Editor:

Recent letters ${ }^{1}$ have stressed fine points in the sophisticated art of awake fiberoptic endotracheal intubation. Our own experience leads us to report several modifications of established technique, which we believe offer useful alternatives in certain clinical settings.

A 30-year-old female with severe rheumatoid arthritis presented for total shoulder arthroplasty under general anaesthesia. A previous attempt under regional anaesthesia had been abandoned because of inadequate block. A difficult intubation was anticipated, because of temperomandibular joint involvement, and decreased neck mobility. Awake fiberoptic intubation was planned. Surgery was once again cancelled, however, because although the bronchoscope could be passed orally into the trachea, a $7.0 \mathrm{~mm}$ PVC endotracheal tube could not be advanced past the lower pharynx.

Two factors were deemed responsible for failure. First, the patient had a hyperactive gag reflex. Second, indirect laryngoscopy the next day suggested that the tip of the endotracheal tube may have been impinging upon a protruding cervical vertebral body displaced anteriorly.

A third procedure was planned. Premedication included intramuscular morphine and hyoscine, and intravenous diazepam. Topical anaesthesia was achieved with a four per cent lidocaine gargle, and intranasal five per cent cocaine. Bilateral superior laryngeal and glossopharyngeal nerve blocks were performed using standard techniques. ${ }^{2,3}$ The cricothyroid membrane was punctured with a $20 \mathrm{~g} \mathrm{IV}$ catheter, and $3 \mathrm{ml}$ of four per cent lidocaine injected. Fiberoptic nasal intubation was successfully undertaken with a $7.5 \mathrm{~mm}$ armoured endotracheal tube. The connector had been removed to allow passage of the bronchoscope, and was replaced after the bronchoscope was withdrawn at the end of the procedure.
Three points are emphasized:

1 The glossopharyngeal nerve block diminished the gag reflex.

2 The flexibility and softness of the armoured tube allowed unimpeded passage through the pharynx into the trachea.

3 Use of a $20 \mathrm{~g}$ IV catheter to puncture the cricothyroid membrane allowed rapid injection of local anaesthetic without the fear of lacerating the larynx or trachea during vigorous coughing.

Donald Oxorn MD FRCPC

Gordon Whatley MD FRCPC

Department of Anaesthesia and Intensive Care Halifax Infirmary

Halifax, Nova Scotia, B3J 2H6

\section{REFERENCES}

1 Green $G G$. Improved technique for fiberoptic intubation. Anesthesiology 1986; 64: 835 .

2 Gotta AW, Sullivan CA. Anaesthesia of the upper airway using topical anaesthetic and superior laryngeal nerve block. Br J Anaesth 1981; 53: 1055-8.

3 Eriksson $E$. Illustrated Handbook in Local Anaesthesia, Ed 2, Copenhagen, Schultz Forlag, 1979

\section{REPLY}

Oxorn and Whatley's comments about a 30-year-old patient with severe rheumatoid arthritis mentioned further options when fiberoptic intubation does not proceed smoothly because of difficulty advancing the endotracheal tube. In such cases, it may be helpful to withdraw the tube a few centimeters, and readvance while rotating the tube in order to orient the tip away from the obstruction. Airway anatomy should be carefully examined as the bronchoscope is passed initially. Abnormal obstructions, such as a protruding cervical vetebral body will be noted. This allows better planning of the actual endotracheal tube passage and a better immediate understanding of problems which may arise as the tube is passed.

Adequate airway anaesthesia is always a prerequisite for an adequate endoscopic examination or elective intubation. We have had excellent success using two per cent lidocaine. The lidocaine solution is placed into the naris and the patient is encouraged to swallow any excess. This provides partial pharyngeal anaesthesia. The bronchoscope is then advanced into the pharynx and additional two per cent lidocaine is injected through the 